\title{
PIXEL-CLUSTER COUNTING LUMINOSITY MEASUREMENT IN ATLAS
}

\author{
William McCormack* \\ on behalf of the ATLAS Collaboration \\ Lawrence Berkeley National Lab. (US) \\ E-mail: william.patrick.mc.cormack.iiiecern.ch
}

\begin{abstract}
A precision measurement of the delivered luminosity is a key component of the ATLAS physics program at the Large Hadron Collider (LHC). A fundamental ingredient of the strategy to control the systematic uncertainties affecting the absolute luminosity has been to compare the measurements of several luminometers, most of which use more than one counting technique. The level of consistency across the various methods provides valuable cross-checks as well as an estimate of the detector-related systematic uncertainties.

This poster describes the development of a luminosity algorithm based on pixel-cluster counting in the recently installed ATLAS inner b-layer (IBL), using data recorded during the $2015 \mathrm{pp}$ run at the LHC. The noise and background contamination of the luminosity-associated cluster count is minimized by a multi-component fit to the measured cluster-size distribution in the forward pixel modules of the IBL. The linearity, long-term stability and statistical precision of the clustercounting method are characterized by comparison with several other ATLAS luminometers.
\end{abstract}

38th International Conference on High Energy Physics

3-10 August 2016

Chicago, USA

${ }^{*}$ Speaker. 
We present a new precision method of determining the LHC luminosity by pixel-cluster counting (PCC) in the most-forward modules of the Insertable b-Layer (IBL) of the ATLAS detector $[1,2]$. These forward modules use 3-D sensors, but that is not relevant to this method. "Pixel clusters" are groups of adjacent hit pixels in the IBL modules. If each cluster corresponds to one primary particle, one can create a luminosity algorithm by counting the pixel clusters. The origins of this project lie in providing a consistency check with the ATLAS tracking-based luminosity monitoring algorithm, which uses distinct components of the ATLAS silicon pixel detector. Further, this method can be applied as a consistency check with CMS, as CMS uses PCC as its baseline luminosity monitoring algorithm [3]. The method described here is based on fitting cluster-length distributions, allowing one to statistically separate the clusters resulting from primary particles (signal) from clusters due to other sources (background) including secondaries from detector material, beam backgrounds, and non-collision backgrounds such as afterglow (out-of-time hits from radiologically activated detector material). Figure 1 shows an example cluster-length distribution within a module with a two-component fit consisting of a Gaussian-shaped signal and an exponentially falling background. By using only modules at the extremes of the IBL and therefore about $30 \mathrm{~cm}$ away from the interaction point, there is an appreciable separation between the signal and background regions due to the low angle of primary particles. We have shown that the signal area of the cluster distribution scales linearly with luminosity, after correcting for inefficient/hot modules and beam spot position and width. For the particular case of afterglow background, a 25ns ATLAS run was dedicated to study this effect, and figure 2 shows the fit components of the cluster-length distribution for a bunch crossing with collisions and subsequent empty crossings.

Several effects must be corrected for in order to convert the signal area to luminosity:

1. Module performance issues (for example a module being disabled for part of a run or a module being noisy) can introduce a bias. We use a data driven method to remove this potential bias. We fit the distribution of signal areas for all IBL 3-D modules at the same position along the beam line to a sinusoidal curve with normalization plus two parameters corresponding to the horizontal and vertical displacement of the beam spot relative to the IBL center. Rather than adding the individual module signal areas, we exclude outliers, re-fit, and use the sinusoidal normalization as the corrected sum of the module signal areas. Figure 3 illustrates such a fit.

2. If the luminous region is not longitudinally centered in the IBL, a forward-backward asymmetry appears in the signal areas. This asymmetry can be used to calculate the longitudinal position of the beamspot through a linear transformation. Figure 4 demonstrates the agreement between the asymmetry-derived position and the ATLAS off-line calculation. Because the beamspot position influences module acceptance nonlinearly, the total signal area (sum of all 3D modules from both sides) will change as the beam spot moves. We correct for this purely geometric effect using a quadratic function of the asymmetry-derived beamspot position. Thus this correction is also data-driven, requiring no external information. Figure 5 shows the influence of beamspot position on acceptance for the forward and backward modules and for all modules considered. 
3. Even if the beam spot center does not move, a longer luminous region will produce a greater signal area, again due to geometric acceptance. The correction for luminous length uses a linear function of the beam spot width obtained from track reconstruction. Figure 6 illustrates the correlation between beamspot length and signal-region clusters.

After corrections the measured luminosity is proportional to

$$
L \propto \frac{N_{s}}{\left(1+C_{1} * R_{o}^{2}+C_{2} * \sigma_{z}\right)}
$$

where $N_{s}$ is the normalization of the sinusoidal to signal areas as in Figure $3, R_{o}$ is the "offset ratio" as in Figure $4, \sigma_{z}$ is the beam spot width, and $C_{1}$ and $C_{2}$ are geometric constants that can be determined from simulation.

Luminosity algorithms must be stable as a function of both time and pileup. Our validation of these conditions relies on comparison of different algorithms using different subdetectors and therefore different sources of potential bias. Figures 7 and 8 compare the new PCC algorithm to the luminometers currently used by ATLAS. The PCC luminosity measurement is stable with respect to the other ATLAS measurements to within $\pm 1 \%$ or better, and is therefore shown to be a competitive relative-luminosity monitoring method.

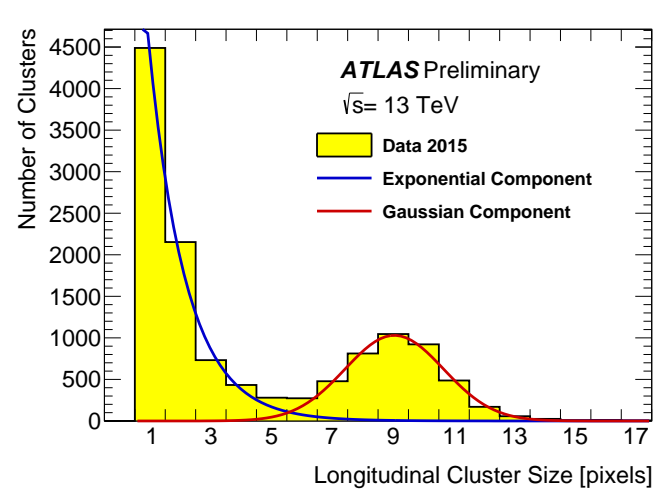

Figure 1: Example of the two component fit to the cluster-length distribution from a single module [4].

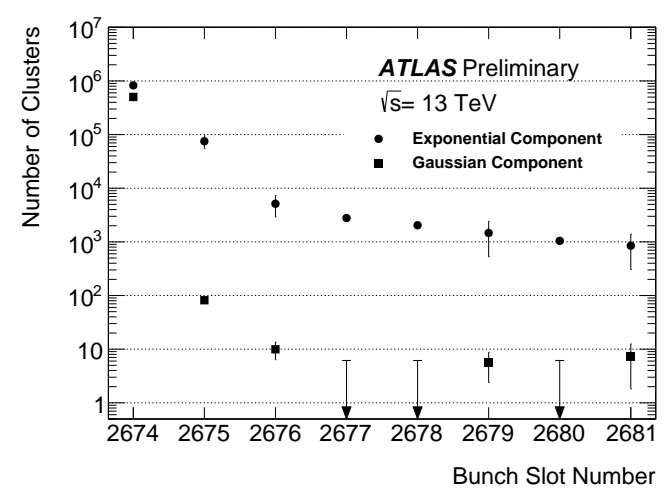

Figure 2: Illustration of afterglow in the IBL: signal-region clusters are represented by squares and background clusters by circles. Proton collisions only occurred in bunch crossing 2674 [4].

\section{References}

[1] ATLAS Collaboration. The ATLAS Experiment at the CERN Large Hadron Collider. JINST, 3:S08003, 2008.

[2] M Capeans et al. ATLAS Insertable B-Layer Technical Design Report.

https://cds.cern.ch/record/1291633.

[3] CMS Collaboration. CMS Luminosity Based on Pixel Cluster Counting - Summer 2013 Update. http://cds.cern.ch/record/1598864.

[4] W McCormack. Pixel-cluster counting luminosity measurement in ATLAS plots for approval. https://cds.cern.ch/record/2200361. 


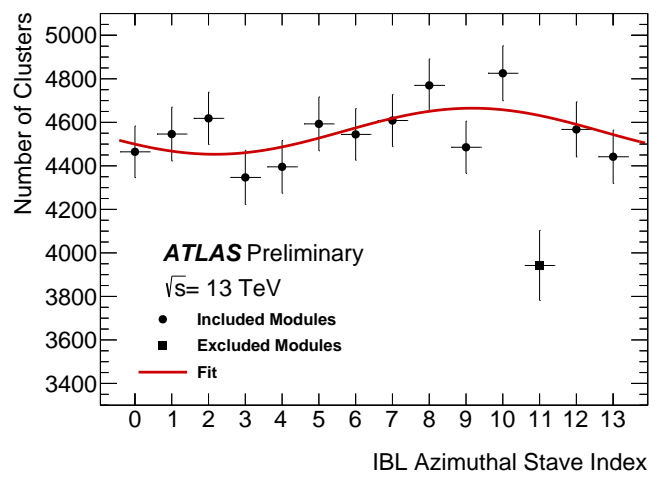

Figure 3: Sample distributions of signal clusters in an IBL ring. The 11th module was excluded [4].

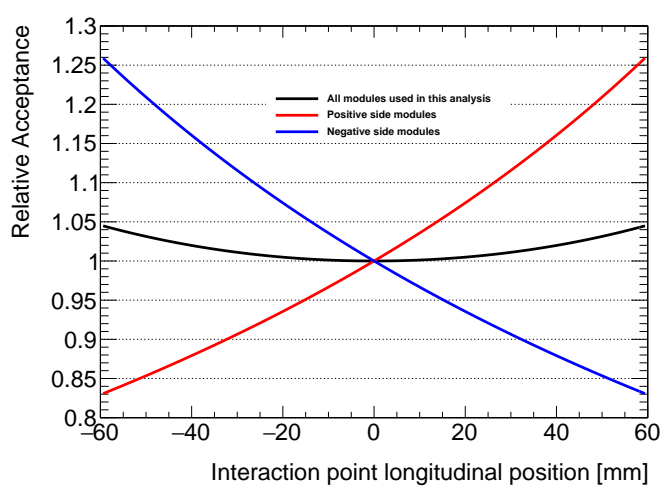

Figure 5: Acceptance correction to the signal region in the modules used by the PCC algorithm as a function of the beamspot position.

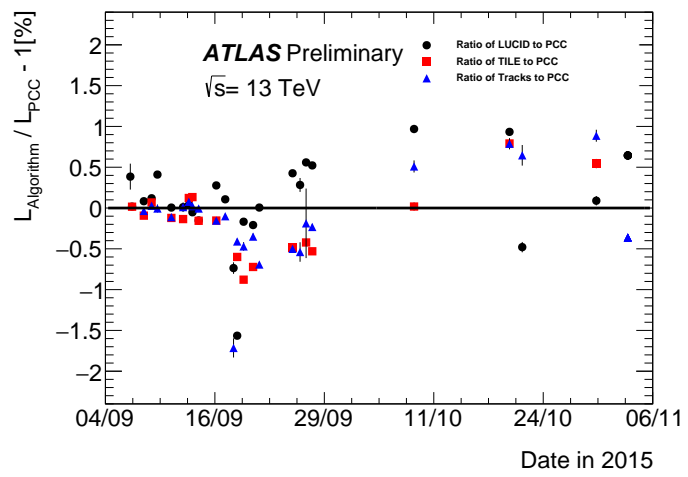

Figure 7: Ratio of PCC-based luminosity to that from other ATLAS luminometers as a function of time during 2015 [4].

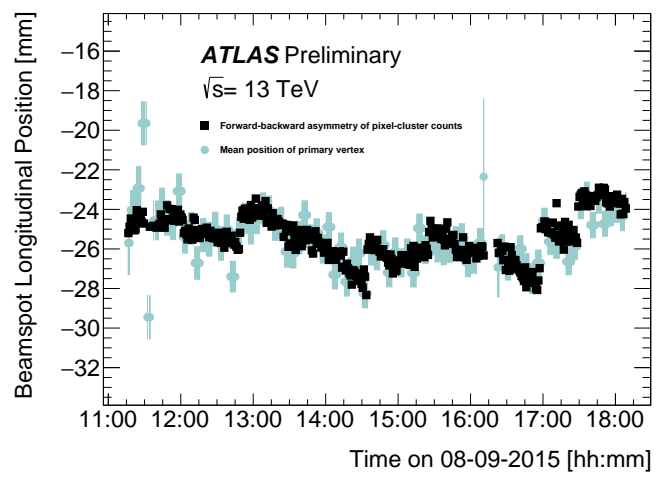

Figure 4: Illustration of the off-line beamspot position calculation (blue) and of the position derived with pixel-cluster asymmetry (black) [4].

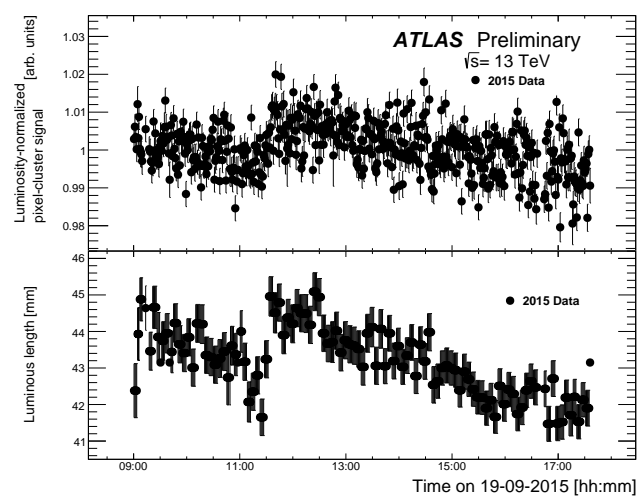

Figure 6: (Above) Normalized number of signalregion clusters as a function of time for a single run. (Below) Beamspot length as a function of time for the same run [4].

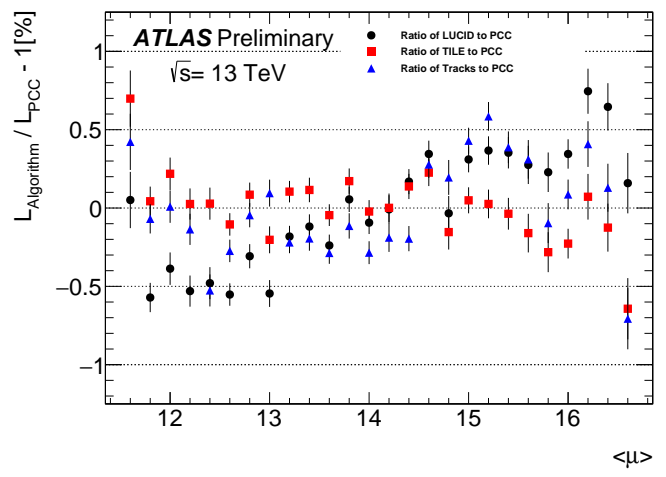

Figure 8: Fractional deviation of bunch-averaged pile-up parameter from the PCC value obtained using different algorithms during a physics run on September 25, 2015 [4]. 